\title{
Histoire de l'énergie marémotrice en France
}

\author{
par M. Banal
}

\section{I 口 LE MOYEN-ÂGE - LES MOULINS A MARÉE}

Il est difficile de fixer la date exacte d'apparition des premiers moulins utilisant l'énergie de la marée pour moudre le blé et il a été dit qu'ils étaient connus du Monde Arabe vers l'an 1000. Mais il est certain que c'est du XII'̀me siècle qu'ils se sont développés sur les côtes françaises de l'Atlantique et de la Manche. Claude Rivals dans le Magazine de l'Histoire $\mathrm{N}^{\circ} 42$, février 1982 , évalue leur nombre à une centaine dont la moitié environ sur les côtes Bretonnes et en particulier dans le golfe du Morbihan où les échancrures nombreuses et les marées relativement fortes conviennent à ces installations. Ils ont été décrits parfaitement par Jacques Guillet dans un article de la revue La Chasse-Marée $\mathrm{N}^{\circ} 5$ 1982 d'après l'état dans lequel ces installations se trouvent actuellement, certains n'ayant été abandonnés que très récemment.

Ici, nous nous contenterons de rappeler que les moulins à marée fonctionnent, sauf exception, au simple effet vidange ; ils comprennent donc une digue, des vannes, une roue extérieure ou intérieure entraînant des meules dans un bâtiment.

Les moulins à marée ont été exploités jusqu'à ce que l'électricité se soit substituée à l'énergie de la marée.

Belidor au XVIII'me siècle, a fait d'importantes études sur les moulins à marée et propose de les faire fonctionner au double effet.

\section{LES ANNÉES AYANT SUIVI LA PREMIÈRE GUERRE MONDIALE}

Du point de vue énergétique, la guerre 14 - 18 a été marquée par une hausse terrible du prix du charbon, le développement des applications de l'électricité (notamment électrification des chemins de fer) et l'affirmation de la vocation hydroélectrique de la France. Cette circonstance a probablement motivé l'intérêt manifesté, dans les différentes occasions énumérées ci-dessous, à la production d'électricité en utilisant l'énergie de marées.

a) Création en 1918 d'une commission de la «Houille Bleue». C'est semble-t-il la première apparition de cette expression pour désigner l'énergie des marées. Cette commission a été animée par les Inspecteurs généraux des Ponts et Chaussées de la Brosse puis Gervais de Rouville.

b) Publication par G. BOISNIER en 1921 d'une étude remarquable sur l'utilisation de l'énergie des marées qui évoque tous les problèmes qui seront étudiés après 1940
L'auteur recommande en particulier l'aménagement de l'estuaire de la Rance.

Il conclut en ces termes :

«En attendant que les conditions économiques aient rendu nécessaire l'utilisation des faibles marées, de celle de la Rochelle et de Brest, c'est par la région de St-Malo qu'il faut commencer et dans cette région, c'est la Rance qui, en raison des conditions exceptionnelles dans lesquelles elle se présente, est tout indiquée pour être la première grande usine marémotrice».

c) Communication sur la «Houille Bleue» au troisième congrès de la Houille Blanche tenu à Grenoble en juillet 1925 présentée par A. de Rouville qui concluait en souhaitant que les études soient poursuivies afin de se trouver prêt le moment venu à utiliser une source d'énergie susceptible de suppléer à d'autres devenues insuffisantes ou relativement moins avantageuses.

d) Le projet de l'Aber-Vrac'h (estuaire situé au nord de Brest) fut présenté au congrès de la Houille Blanche de 1925.

Cette usine marémotrice avait été choisie par les pouvoirs publics comme une station expérimentale préparant la réalisation de l'usine de la Rance.

Bien qu'ayant fait l'objet d'une concession de forces hydrauliques approuvée le 22 août 1924, ce projet ne reçut aucun début de réalisation.

\section{III घ'ÉNERGIE MARÉMOTRICE EN FRANCE APRÈS LA SECONDE GUERRE MONDIALE}

En 1940, Robert Gibrat alors Directeur de l'électricité au ministère des Travaux Publics découvrit l'Energie marémotrice en consultant le livre : L'utilisation de l'Energie de Marées de G. Boisnier, Ingénieur des Ponts et Chaussées à Rennes datant de 1921.

Cette étude pourtant très remarquable n'avait reçu à l'époque aucune application concrète comme nous l'avons dit au chapitre II, mais Robert Gibrat pensa que les progrès intervenus dans les techniques étaient susceptibles de rendre réalisable ce qui ne l'avait pas paru alors.

En 1941 à son initiative, des sociétés intéressées par la production d'électricité créèrent la Société d'étude pour l'utilisation des marées (S.E.U.M) dont Robert Gibrat était l'animateur et qui engagea immédiatement un programme d'études. C'est ainsi qu'un modèle réduit de l'estuaire de la Rance fut réalisé 
au Laboratoire Dauphinois d'Hydraulique et la société Neyrpic se vit confier des recherches sur les turbines de très basse chute utilisables pour les usines marémotrices.

A la nationalisation de l'électricité en 1946, l'Electricité de France (E.D.F.) reprit les activités de la société d'études pour l'utilisation des marées dans un service aux mêmes initiales et Robert Gibrat devient Ingénieur-Conseil d'E.D.F. pour les usines marémotrices.

Si les perspectives d'utilisation de l'énergie marémotrice s'étaient limitées à l'estuaire de la Rance avec une production d'environ 500 millions de francs, Electricité de France n'aurait peut-être pas développé les études nécessaires. Aussi, le S E U M se vit-il confier aussi les études d'un aménagement de beaucoup plus grande taille utilisant l'énergie de la marée.

Il y avait de nombreuses possibilités de créer des réservoirs dans la région comprise entre l'ouest du Cotentin et la côte de Bretagne où l'amplitude de la marée est exceptionnelle, mais assez rapidement le choix s'arrêta sur un projet de Chausey dont le bassin était obtenu en reliant l'archipel du même nom à la côte du Cotentin un peu au nord de Granville et à la pointe du Grouin.

La production attendue de ce projet était 24 milliards de $\mathrm{kWh}$ soit cinquante fois environ celle de l'usine de la Rance.

Les études poursuivies par le S E U M pour mettre au point le projet de la Rance étaient évidemment utiles au projet de Chausey mais a contrario l'étude de celui-ci posait des problèmes spécifiques en ce qui concerne notamment la compréhension et la représentation sur modèle des phénomènes de marée dans des surfaces maritimes importantes.

Pour ces études, le S E U M disposait de la coopération des autres services d'E.D.F. et notamment de la Direction des Etudes et Recherches mais aussi des organismes de recherche publics et des constructeurs de matériels.

Le projet de la Rance posait deux problèmes principaux, non indépendants d'ailleurs, le ou les cycles de fonctionnement et les caractéristiques des groupes et leur protection contre la corrosion de l'eau de mer.

Assez rapidement, les groupes bulbes étudiés notamment par la société Neyrpic furent adoptés et E.D.F. commanda en 1955 quatre groupes expérimentaux de ce type à installer dans les centrales hydroélectriques d'Argentat, Cambayrac et Beaumont-Monteux puis sans attendre la mise en service de ces groupes et avec l'appui du Ministère des Travaux Publics, E.D.F. décida d'installer un groupe bulbe aux caractéristiques identiques à celles projetées pour la Rance dans une écluse désaffectée de St-Malo. Ce groupe fut mis en service en 1959 et les enseignements qui en furent tirés, furent d'un grand poids dans la décision des pouvoirs publics approuvant le projet présenté par E.D.F. pour l'aménagement de la Rance.

En 1951, les études n'étaient certes pas achevées mais considérées par E.D.F. comme assez avancées pour dresser un premier projet qui permit d'engager la procédure administrative et notamment la demande en concession dont l'instruction se déroula comme indiqué ci-après :

- le 26 mars 1952, dépôt par E.D.F. d'une demande de concession de l'Usine de la Rance avec déclaration d'utilité publique en application de la loi du 16/10/19,

- le 25 octobre 1952, le Président de la République Vincent Auriol, lors de l'inauguration de l'usine de Donzère Mondragon annonçait l'aménagement prochain de l'usine de la Rance. - L'enquête et les conférences règlementaires furent ouvertes en 1953 et donnèrent dans l'ensemble des résultats très favorables.

- Par la suite, E.D.F. déposa un dossier complémentaire pour tenir compte des dernières dispositions techniques adoptées.

- Par décision du 12 juin 1956, l'Ingénieur en Chef de la 3 ème circonscription électrique procéda à la clôture de l'instruction prévue par les textes règlementaires en matière de concession de force hydraulique. Cependant, la loi du 15/01/18 interdisant sauf autorisation donnée par le Parlement la construction d'un barrage dans la partie maritime navigable des fleuves et rivières dont la liste est annexée à la loi (La Rance en faisait partie) une loi spéciale était donc nécessaire. Elle fut votée le 8 août 1956 et autorisa le barrage de l'estuaire de la Rance dans sa partie maritime.

— Par ailleurs, la loi du 27 mars 1956 approuvant le 2ème plan de modernisation et d'équipement avait prévu dans le programme hydraulique l'engagement de l'usine marémotrice de la Rance qui se trouva ainsi deux fois approuvée par le Parlement.

En 1960, le dernier projet de l'usine de la Rance fut arrêté par la région d'équipement marémotrice spécialement créée à cet effet en 1951 et soumis aux pouvoirs publics. Le 29 décembre 1960, le ministère de l'Industrie autorisa E.D.F. à engager un programme d'aménagement hydroélectrique comprenant la Rance.

Les premiers chantiers furent ouverts début 1961 et en juillet 1963 la Rance était coupée. L'usine fut inaugurée par le Général de Gaulle, Président de la République en 1966 et le 24ème groupe était mis en service le 4 décembre 1967.

En 1967, la région d'équipement marémotrice disparut et l'entretien et l'exploitation de l'usine intégralement confiés au service de la Production Hydraulique (G R P H Massif Central puis G M E H T Bretagne créé en 1971). Ce fut le service de la production hydraulique qui remédia aux maladies de jeunesse des groupes et spécialement des alternateurs. Elle réalisa aussi l'automatisation complète de l'exploitation de l'usine en fonction de la marée et des besoins du réseau en tenant compte de l'expérience acquise et des progrès de l'informatique.

En 1980, les études de réalisation d'un grand aménagement marémoteur furent reprises en mettant l'accent sur les trois points suivants :

- Le coût,

- La valeur de la production,

- L'effet sur l'environnement.

Ces études furent complètement arrêtées en 1983, leurs perspectives n'ayant pas paru suffisamment prometteuses.

A partir de cette date, la compétence de la France dans l'énergie marémotrice ne s'accroît plus que de l'expérience de l'usine de la Rance et ce n'est pas rien... 